\title{
El papel del agua sobre la biomasa vegetal en la zona semiárida con clima mediterráneo de Chile ${ }^{1}$
}

\author{
Javier Lozano-Parra², Carlos Lozano-Fondón³, Manuel Pulido ${ }^{4}$ \\ y Ramón García-Marín ${ }^{5}$
}

\begin{abstract}
RESUMEN
Los ecosistemas semiáridos mediterráneos de Chile soportan altas tasas demográficas y mantienen importantes economías asociadas a la productividad del medio natural, sin embargo, dependen fuertemente de la disponibilidad hídrica del medio. A pesar de ello, los estudios desarrollados en ambientes limitados por el agua abordados desde un enfoque ecohidrológico son todavía muy escasos en la zona semiárida de Chile. Este estudio intenta definir el papel del agua sobre el crecimiento de la biomasa herbácea utilizando una combinación de instrumentos que permitieron monitorizar con gran resolución temporal la dinámica vegetal y distintas variables asociadas al ciclo hidrológico. Los resultados mostraron que entre el $57 \%$ y el $93 \%$ de la varianza en la dinámica vegetal puede ser explicada utilizando únicamente tres variables asociadas al ciclo hidrológico (precipitación, evapotranspiración y contenido hídrico del suelo). De este modo, un cambio en dicho ciclo podría amenazar el mantenimiento y productividad de estos ecosistemas.
\end{abstract}

Palabras clave: Ecohidrología; agua; vegetación; NDVI; ecosistemas semiáridos.

\begin{abstract}
The semi-arid Mediterranean ecosystems of Chile sustain high demographic rates and maintain very important economies related to the environmental productivity, although they strongly depend on water availability. Nevertheless, studies carried out on water-limited environments and from an ecohydrological framework, are very scarce in the semi-arid areas of Chile. This study tries to define the role of water on herbaceous biomass growth using equipment which monitored some key variables concerning to the water cycle and biomass dynamics with a high temporal resolution. Results showed that between $57 \%$ and $93 \%$ of total variance in biomass dynamics could be explained using only three variables related to hydrological cycle, such as rainfall, evapotranspiration and soil water content. We concluded that a change in the water cycle of these areas could threat the productivity and maintenance of these ecosystems.
\end{abstract}

Keywords: Ecohydrology; water; vegetation; NDVI; semiarid ecosystems.

El presente trabajo ha sido desarrollado gracias a la financiación de los proyectos FONDECYT 11161097 y REDI170640, ambos concedidos por la Comisión Nacional de Investigación Científica y Tecnológica (CONICYT) de Chile. Artículo recibido el 30 de diciembre de 2017, aceptado el 30 de mayo de 2018 y corregido el 30 de agosto de 2018.

Instituto de Geografía, Pontificia Universidad Católica de Chile (Chile). E-mail: jlozano@outlook.es

Departamento de Química, Ciencias de la Vida y Sostenibilidad Ambiental, Universidad de Parma (Italia). E-mail: Izncls@unife.it

Grupo de Investigación GeoAmbiental, Universidad de Extremadura (España).E-mail: mapulidof@unex.es

Departamento de Geografía, Universidad de Murcia (España). E-mail: ramongm@um.es 
Alrededor del $41 \%$ de la superficie global está compuesta por tierras áridas, semiáridas o subhúmedas secas (Reynolds et al., 2007). Entre ellas se encuentran varios tipos de ecosistemas con estructura forestal de arbolado disperso, como las sabanas tropicales africanas, los rangelands californianos, las dehesas ibéricas, o el espinal mediterráneo del territorio central de Chile, ampliamente establecido entre la IV y VIII Región, y con una extensión estimada de unas 4 millones de hectáreas (Ovalle et al., 1990).

Los espinales son sistemas silvopastoriles que deben ser conservados, ya que normalmente soportan altos niveles de biodiversidad dentro de una amplia variedad de ambientes, como áreas de forrajes o de pastizal, espacios de pastoreo o zonas de matorral. Además, mantienen importantes economías asociadas a la explotación del ecosistema (pastoreo, agricultura, extracción de cortezas, miel, provisión de forrajes, etc.) y, consecuentemente, a la productividad del medio natural (Root-Bernstein \& Jaksic, 2013). Sin embargo, su equilibrio y mantenimiento es delicado debido a que dos de los factores que determinan su configuración, la gestión humana y las condiciones físico-ambientales, ejercen un importante control sobre sus componentes clave, el agua y la biomasa vegetal.

Los ecosistemas de espinal con clima mediterráneo poseen unas condiciones físico-ambientales caracterizadas por la estacionalidad y escasa predictibilidad de la precipitación, tanto en cantidad como en distribución espacio-temporal, y por las altas temperaturas y duración de la estación seca, lo cual origina una elevada evapotranspiración potencial, que a su vez determina un gran déficit hídrico. De esta forma, la irregularidad climática condiciona la disponibilidad hídrica del suelo, la cual representa el principal limitante del ecosistema (Valladares et al., 2004)2004. Las alteraciones climáticas podrían modificar la dinámica natural de la biomasa, dando lugar a variaciones en la producción que afecten tanto al ecosistema como a las economías dependientes del medio natural (Lozano-Parra et al., 2014).

La biomasa vegetal asociada a las herbáceas anuales constituye uno de los principales recursos en los sistemas silvopastoriles semiáridos de espinal chileno, ya que suponen un eficaz elemento contra la degradación y erosión del suelo, y constituyen la principal fuente de alimentación para el ganado y, consecuentemente, desempeñan un papel clave sobre las economías de estos ambientes (Root-Bernstein \& Jaksic, 2013, Schnabel et al., 2013). La producción de biomasa herbácea depende del balance de efectos positivos o negativos entre cada uno de los principales factores limitantes: agua, energía lumínica y térmica, nutrientes y espacio (Brooker et al., 2008). Sin embargo, los estudios de producción de biomasa frecuentemente han sido desarrollados en Europa, Norteamérica o Australia, siendo poco frecuentes en las zonas semiáridas de espinal chileno (Campos et al., 2013, Rigueiro-Rodríguez et al., 2009). Además, se han enfocado en la competición/facilitación entre estratos vegetales o en la respuesta productiva frente a diferentes tratamientos, restando importancia al funcionamiento de la zona superficial del suelo en condiciones naturales (Brooker et al., 2008, Moreno, 2008, Schnabel et al., 2001).

Por otro lado, los estudios desarrollados en ambientes limitados por el agua que abordan desde una perspectiva combinada los procesos hidrológicos y ecológicos (ecohidrológicos, de aquí en adelante), son todavía escasos y, para conocimiento de los autores, son poco frecuentes en sistemas silvopastoriles de la zona central de Chile. No obstante, en otras zonas del mundo con sistemas similares, como en Europa y Norteamérica, numerosos autores han realizado destacados 
avances en los últimos años en el campo de la hidrología aplicada a dichos sistemas (Cubera, 2006; Maneta, 2006; Sepúlveda Manzor, 2010; Van Schaik, 2010) intentando cubrir con ellos la falta de conocimiento existente y que ya subrayó Campos-Palacín (2004). Sin embargo, el hándicap se encuentra en que no combinan con precisión la resolución temporal junto con la espacial ya que, o bien monitorizan el contenido hídrico del suelo a escalas temporales normalmente superiores a la semanal, o bien eluden medir con precisión la zona más superficial del suelo, de modo que no cubren ciertas escalas espacio-temporales debido a sus limitaciones metodológicas (Lozano-Parra, 2015).

En función de lo expuesto, los objetivos de esta investigación fueron: i) analizar el efecto de las variaciones meteorológicas sobre sobre la dinámica del contenido de agua del suelo bajo diferentes estratos vegetales (árboles y herbáceas), utilizando una alta resolución temporal y monitorizando la principal capa edáfica que influye sobre las herbáceas anuales; y ii) establecer la relación entre el contenido de agua del suelo y la producción de biomasa herbácea aérea durante el periodo de vida de las plantas anuales. Conviene destacar que estudios exhaustivos como el aquí presentado son, para conocimiento de los autores, muy escasos en ambientes mediterráneos de espinal de la zona central de Chile.

\section{Área de estudio}

Los trabajos se desarrollaron en los campos de la Agrícola y Ganadera Corralillo S.A, localizada entre las comunas de Casablanca y Cartagena, pertenecientes a la provincia de Valparaíso y a la región homónima (Figura $N^{\circ} 1$ ). Se trata de una zona con aproximadamente 16.000 hectáreas propiedad de la firma Viña Matetic, caracterizada por ser representativa de los ecosistemas mediterráneos semiáridos de la zona central de Chile.

El clima se caracteriza por ser mediterráneo de lluvia invernal (Csb, según la clasificación climática de Köppen-Geiger, en: Rubel \& Kottek, 2010), con precipitaciones medias inferiores a los $700 \mathrm{~mm}$ y concentradas en invierno debido a la influencia oceánica, y temperaturas medias en torno a los $11^{\circ} \mathrm{C}$. Los veranos son secos y cálidos, los inviernos lluviosos y templados, y las estaciones transitorias son variables, tanto en temperaturas como en precipitaciones.

La vegetación está compuesta principalmente por especies características de la zona semiárida, como la Acacia caven y Prosopis chilensis, y por un estrato herbáceo formado por praderas antiguas naturalizadas, donde predomina el género Phalaris. El arbolado se encuentra disperso y muestra una densidad media-baja (25-50\%), dando lugar a una estructura forestal de tipo sabanoide, denominado espinal, que representa el $90 \%$ de lo que localmente se conoce como secano interior (Ovalle et al., 1990). El principal uso de suelo es ganadero de tipo extensivo, aunque en otros sectores la actividad predominante es la viticultura. Se trata de un tipo de ecosistema similar a los encontrados en otras regiones mediterráneas del mundo, como las dehesas/montados de la Península Ibérica o los rangelands californianos.

Los suelos se clasifican principalmente como Luvisoles y, en menor medida, como Calcisoles, según la HWSD (FAO, 2012). Ambos pueden ser considerados como arcillo-arenosos, y presentan las características que se resumen en el Cuadro $\mathrm{N}^{\circ} 1$. 
Figura $\mathrm{N}^{\circ} 1$

Localización del área de estudio
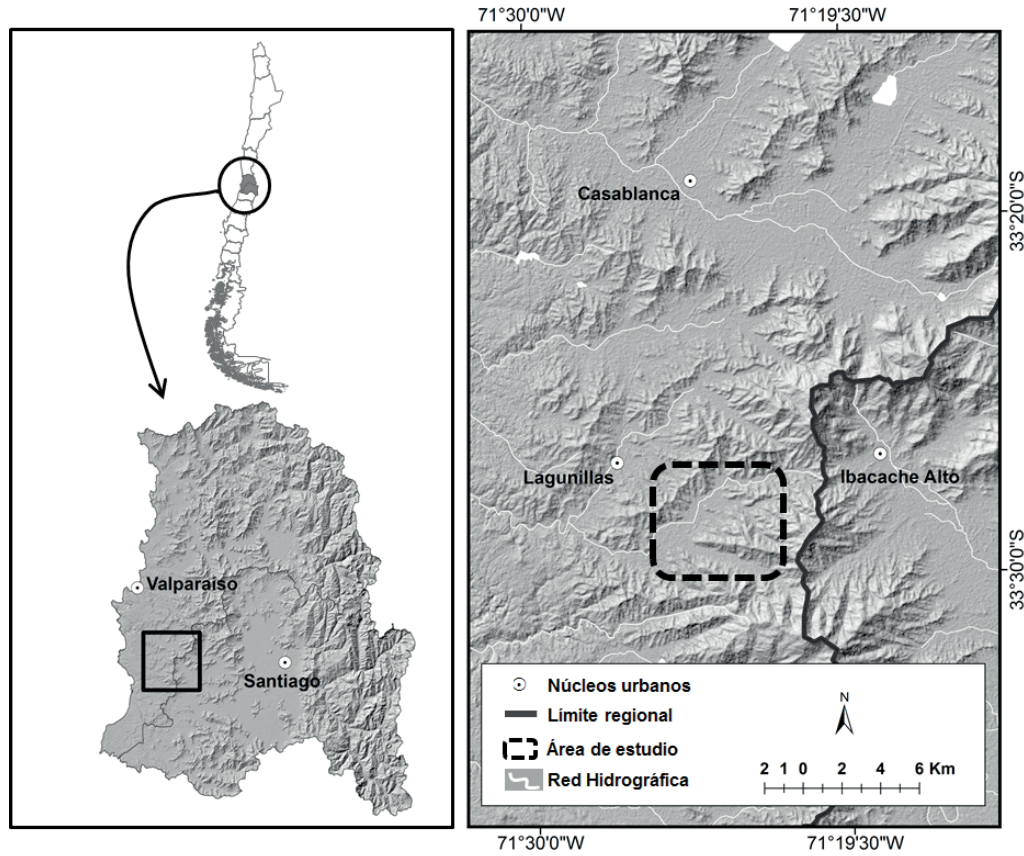

Fuente: Elaboración propia.

\section{Cuadro $N^{\circ} 1$}

Propiedades físicas de los suelos del área de estudio. C.O = Carbono orgánico (\% del peso);

D. $A=$ Densidad aparente $\left(\mathrm{g} / \mathrm{cm}^{3}\right)$

\begin{tabular}{|l|r|r|r|r|r|r|}
\hline \multirow{2}{*}{ Suelo } & $\begin{array}{r}\text { Potencia } \\
(\mathrm{cm})\end{array}$ & \multicolumn{1}{c|}{ \% Arena } & \% Limo & \% Arcilla & \% C.O. & \multicolumn{1}{c|}{ D.A. } \\
\hline \multirow{2}{*}{ Luvisol } & $0-30$ & 51 & 22 & 27 & 0.63 & 1.38 \\
\cline { 2 - 7 } & $30-100$ & 45 & 21 & 34 & 0.35 & 1.33 \\
\hline \multirow{2}{*}{ Calcisol } & $0-30$ & 54 & 24 & 22 & 0.40 & 1.42 \\
\cline { 2 - 7 } & $30-100$ & 47 & 24 & 29 & 0.20 & 1.36 \\
\hline
\end{tabular}

Fuente: Elaboración propia a partir de la información facilitada por la HWSD (FAO, 2012).

\section{Material y Métodos}

\section{Contenido hídrico y potencial de succión del suelo}

El área de estudio fue equipada con seis estaciones automáticas de medición de la humedad del suelo (EHS) que registraron el contenido volumétrico de agua $\left(\mathrm{m}^{3} \mathrm{~m}^{-3}\right)$ y el potencial hídrico del suelo $(\mathrm{kPa})$ en intervalos de 5 minutos durante el ciclo de vida anual de las herbáceas, es decir, 
considerando el otoño, invierno y primavera del año hidrológico 2017. Cada EHS se compone de cuatro sensores que miden la humedad del suelo $(a, 5,15,25$ y $35 \mathrm{~cm}$ de profundidad) y por un sensor de potencial hídrico a $10 \mathrm{~cm}$ de profundidad (Figura $\mathrm{N}^{\circ} 2 \mathrm{~A}$ ). Este último permite conocer el potencial de succión edáfico al que se encuentran en cada momento los contenidos hídricos monitorizados, destacando entre ellos el contenido de agua del suelo a $-33 \mathrm{kPa}$ y $-1500 \mathrm{kPa}$ (puntos discutiblemente establecidos como capacidad de campo y punto de marchitamiento, que permiten definir el contenido de agua disponible para las plantas). La profundidad de instalación de los sensores se justifica porque el sistema radicular de la mayor parte de las especies herbáceas de estos ecosistemas se concentra en los primeros 30 centímetros del perfil edáfico (Canadell \& Zedler, 1995). Tres EHS fueron instaladas bajo copa de árbol (A) y otras tres en espacios de pastizal $(\mathrm{P})$ o fuera de la influencia del árbol (Figura $\mathrm{N}^{\circ} 2 \mathrm{~B}$ y $2 \mathrm{C}$, respectivamente). Las primeras se instalaron entre el tronco y el borde de la copa, mientras que las segundas en zonas de ladera representativas de las unidades fisiográficas predominantes, evitando las vaguadas o zonas de acumulación hídrica. Cada EHS ha sido cercada y parcelada por un vallado de alambre para protegerla de los efectos del ganado.

Figura $\mathrm{N}^{\circ} 2$

Estación de Humedad del Suelo -EHS- (A); EHS bajo copa de árbol (B); EHS en espacio abierto o de pastizal (C)
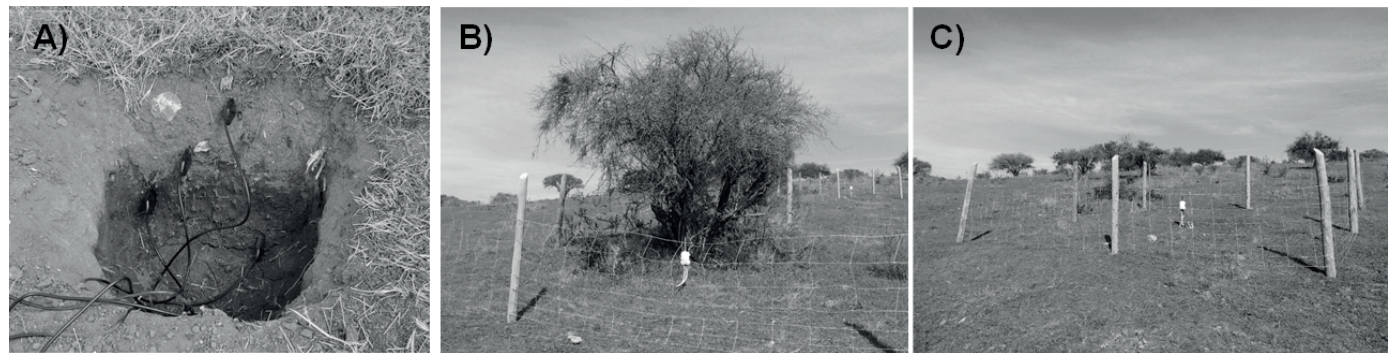

Fuente: Elaboración propia.

\section{Dinámica de la biomasa herbácea}

El crecimiento de la biomasa aérea de las herbáceas se registró durante el ciclo de vida de las plantas anuales, es decir, durante el otoño, invierno y primavera. Para ello, el área de estudio fue equipada con un dispositivo de reflectancia espectral que permitió obtener el Índice Diferencial de Vegetación Normalizado (o Normalized Difference Vegetation Index -NDVI-) siguiendo el siguiente procedimiento:

$$
N D V I=\frac{N I R-R}{N I R+R}
$$

donde, NDVI es el Índice Diferencial de Vegetación Normalizado; NIR es la reflectancia correspondiente al infrarrojo cercano; $\mathrm{R}$ es la reflectancia correspondiente al rojo. 
El NDVI constituye un buen estimador de la fracción de la radiación fotosintéticamente activa interceptada por la vegetación (PAR), del estado de salud y vigorosidad de la vegetación, o de la productividad primaria. Se trata de un índice adimensional, y sus valores oscilan entre -1 y +1 . En sentido práctico, los valores inferiores a 0.1 se corresponden con cuerpos de agua o suelo desnudo, mientras que los valores superiores son indicadores de actividad fotosintética (Chuvieco, 2016). De este modo, el sensor de reflectancia espectral posibilitó la monitorización continua (cada $5 \mathrm{~min}$ ) de la vegetación siguiendo una metodología no destructiva, y permitió registrar el ciclo completo de crecimiento de los pastos anuales para monitorizar su fenología.

Por otro lado, el crecimiento de las herbáceas en altura $(\mathrm{cm})$ fue registrado con una frecuencia quincenal durante todo el ciclo de vida de las plantas anuales, tomando mediciones en 16 puntos sobre $1 \mathrm{~m}^{2}$ de cada EHS. Posteriormente, se promedió un valor representativo a partir de dichos puntos en cada EHS y para cada fecha en la que se registró la altura. De este modo, se pudo establecer la relación entre el NDVI y el crecimiento de la biomasa en altura.

\section{Variables meteorológicas}

Las variables climáticas de precipitación $(\mathrm{mm})$ y temperaturas $\left({ }^{\circ} \mathrm{C}\right)$ fueron monitorizadas mediante un pluviómetro automático (modelo ECRN de Decagon Devices) y un sensor de temperatura ambiental (modelo VP4 de Decagon Devices), que registraron de forma continua y en intervalos de 5 min la pluviometría y las variaciones térmicas. Dichas variables permitieron calcular la evapotranspiración de referencia ( $\mathrm{ET}_{\mathrm{O}}$ ) diaria mediante la ecuación de Hargreaves, siguiendo el método establecido por Allen et al. (1998)

$$
E T_{O}=0.0023\left(T_{\text {media }}+17.8\right)\left(T_{\max }-T_{\min }\right)^{0.5} R_{a}
$$

donde, $T_{\text {media }}, T_{\max }$ y $T_{\text {min }}$ son la temperatura media, máxima y mínima diaria $\left({ }^{\circ} \mathrm{C}\right)$, respectivamente; y $R_{a}$ es la radiación solar extraterrestre diaria $\left(M J m^{-2}\right.$ día $\left.{ }^{-1}\right)$.

Además, con el fin de caracterizar el régimen pluviométrico anual y estacional de la zona donde se enmarca el área de estudio, se utilizaron tres series de precipitación superiores a 30 años procedentes del Centro de Ciencia del Clima y la Resiliencia de Chile, y correspondientes a las estaciones meteorológicas de Casablanca, Lagunillas e Ibacache Alto (Figura $N^{\circ} 1$ ).

\section{Análisis estadísticos}

Con el fin de analizar las diferencias en los contenidos hídricos entre estaciones de humedad del suelo, se aplicó una metodología que permite obtener la estabilidad temporal de la humedad a escala diaria. El test paramétrico de diferencias relativas, propuesto por Vachaud et al. (1985), establece un patrón de comportamiento en un punto de medida con respecto al comportamiento medio del conjunto de puntos a lo largo del tiempo, reflejando la persistencia temporal de la estructura espacial, permitiendo calcular sus diferencias relativas $\left(\delta_{i j}\right)$ y representarlas gráficamente con el fin de destacar similitudes y contrastes (Martínez-Fernández \& Ceballos Barbancho, 2005). Se establece como sigue:

$$
\delta_{i j}=\frac{\Delta_{i j}}{\bar{S}_{j}}
$$


donde,

$$
\Delta_{i j}=S_{i j}-\bar{S}_{j}
$$

y

$$
\bar{S}_{i j}=\frac{1}{N} \sum_{i=1}^{N} S_{i j}
$$

siendo, $\bar{S}_{i j}$ el contenido volumétrico de agua $\left(\mathrm{m}^{3} \mathrm{~m}^{-3}\right)$ en el punto $i$ el día $j$, y $N$ el rango de muestreo.

Posteriormente, la diferencia relativa media $\bar{\delta}_{i}$ puede ser calculada sobre el número total de días considerados, siendo el número total de días de muestreo:

$$
\bar{\delta}_{i}=\frac{1}{m} \sum_{j=1}^{m} \delta_{i j}
$$

La desviación estándar de la diferencia relativa media en cada punto, $\sigma\left(\delta_{i}\right)$, puede ser entonces calculada como un estimador de la variabilidad temporal de la humedad en cada punto:

$$
\sigma\left(\delta_{i}\right)=\sqrt{\frac{\sum_{j=1}^{m}\left(\delta_{i j}-\bar{\delta}_{i}\right)}{m-1}}
$$

Por otro lado, con el objetivo de establecer la relación entre los flujos hídricos (en varios procesos del ciclo del agua, como la precipitación, evapotranspiración, o contenido hídrico del suelo) y el crecimiento de la biomasa herbácea se llevó a cabo un test estadístico no-paramétrico de correlación de Spearman entre las variables asociadas al ciclo hidrológico y la dinámica vegetal. Posteriormente, se realizó un análisis de regresión múltiple con el fin de determinar el efecto combinado de las variables del ciclo hidrológico sobre la dinámica de la biomasa. Dicho análisis ayudó a construir dos modelos del tipo:

$$
y=\alpha+\beta_{1} * X_{1 i}+\beta_{2} * X_{2 i}+\varepsilon_{i}
$$

donde, $y$ es la variable dependiente, $X$ son las variables independientes, i son los números de observaciones, y $\beta$ son los coeficientes de regresión.

Los análisis fueron realizados con Statistica $8^{\odot}$ (StatSoft, Inc.), que implementa las herramientas necesarias que permitieron determinar el papel de las variables hídricas sobre el desarrollo de las herbáceas.

\section{Caracterización del régimen pluviométrico estacional}

El régimen pluviométrico de las tres estaciones meteorológicas analizadas se caracterizó por su variabilidad temporal, tanto anual como estacional, y por su escasa variabilidad en el espacio. 
La variabilidad espacial de la lluvia puede presentar altos contrastes a nivel regional asociados fundamentalmente a la orografía e influencia del océano Pacífico. Sin embargo, la precipitación media anual durante el periodo pluviométrico analizado (1986-2016) presentó valores muy similares entre Lagunillas $(376.8 \mathrm{~mm})$, Casablanca $(376.1 \mathrm{~mm})$ e Ibacache Alto $(369.5 \mathrm{~mm})$, principalmente debido a la cercanía entre estaciones y la escasa diferencia altitudinal entre ellas (169-290 m s.n.m.). A pesar de dicha similitud, la distribución temporal de la precipitación anual se caracterizó por su irregularidad (Figura $\mathrm{N}^{\circ} 3$ ), como era de esperar para el clima mediterráneo, lo que originó frecuentes períodos de sequía alternados con épocas relativamente húmedas (Valdés-Pineda et al., 2014).

Figura $\mathrm{N}^{\circ} 3$

Precipitación anual $(\mathrm{mm})$ en las estaciones meteorológicas de Lagunillas, Casablanca e Ibacache Alto durante el periodo 1986-2016. Las líneas horizontales representan la precipitación media obtenida entre las tres estaciones meteorológicas más/menos su desviación estándar

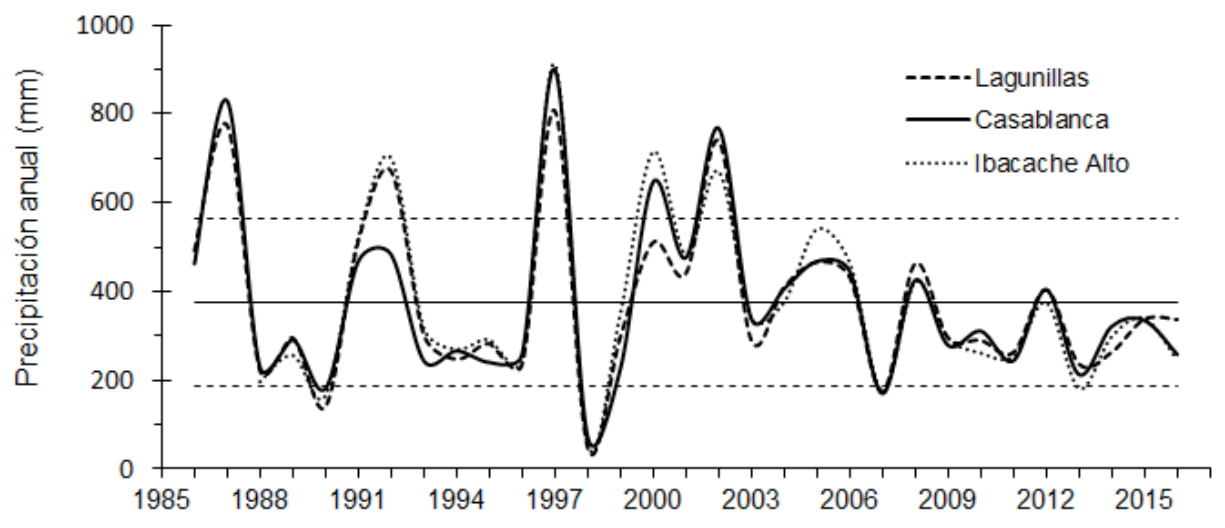

Fuente: Elaboración propia.

La pluviometría estacional media de las tres estaciones meteorológicas se concentró en invierno, entre los meses de junio y agosto, con valores próximos a los $245 \mathrm{~mm}$, mientras que el otoño y la primavera constituyeron periodos menos lluviosos, con valores medios próximos a los $80 \mathrm{~mm}$ y $44 \mathrm{~mm}$, respectivamente (Cuadro $N^{\circ} 2$ ). La pluviosidad estival fue prácticamente inexistente, con una habitual ausencia de precipitaciones entre diciembre y mediados de abril, siendo frecuente la acumulación de 90 días, o más, sin precipitación. Las lluvias que se produjeron durante esta época tuvieron origen convectivo y normalmente se caracterizaron por su intensidad y por su carácter local (Garreaud et al., 2009). La pluviometría estacional observada en el área de estudio durante el año 2017 presentó valores similares a los promedios registrados en las tres estaciones meteorológicas. En invierno se alcanzaron $248 \mathrm{~mm}$, en otoño $95 \mathrm{~mm}$, mientras que los valores de primavera fueron algo más elevados que su promedio, con $79 \mathrm{~mm}$. De esta forma, si se compara con las series de precipitación de 30 años de las estaciones meteorológicas circundantes, el régimen pluviométrico estacional para la zona y el periodo estudiado podría considerarse como normal. 
Cuadro $\mathrm{N}^{\circ} 2$

Precipitación estacional media $(\mathrm{mm})$ en las estaciones meteorológicas de Lagunillas, Casablanca e Ibacache Alto durante el periodo 1986-2016.Prom. = media; D.E = desviación estándar. Otoño = Mar.,

Abr., May.; Invierno = Jun., Jul., Ago.; Primavera = Sep., Oct., Nov.; Verano = Dic., Ene., Feb.

\begin{tabular}{|l|r|r|r|r|r|r|r|r|}
\hline & \multicolumn{2}{|c|}{ Otoño } & \multicolumn{2}{c|}{ Invierno } & \multicolumn{2}{c|}{ Primavera } & \multicolumn{2}{c|}{ Verano } \\
\hline & Prom. & \multicolumn{1}{c|}{ D.E } & \multicolumn{1}{c|}{ Prom. } & \multicolumn{1}{c|}{ D.E } & \multicolumn{1}{c|}{ Prom. } & \multicolumn{1}{c|}{ D.E } & \multicolumn{1}{c|}{ Prom. } & \multicolumn{1}{c|}{ D.E } \\
\hline Lagunillas & 83,8 & 77,3 & 247,5 & 132,2 & 41,7 & 39,8 & 3,2 & 9,8 \\
\hline Casablanca & 84,3 & 80,6 & 243,0 & 13,7 & 45,0 & 48,2 & 3,3 & 8,6 \\
\hline Ibacache A. & 79,0 & 72,1 & 243,0 & 131,4 & 44,0 & 49,7 & 3,4 & 8,4 \\
\hline
\end{tabular}

Fuente: Elaboración propia.

\section{Evolución temporal de los flujos hídricos}

Las series temporales de humedad del suelo, precipitaciones y evapotranspiración mostraron patrones estacionales asociados principalmente a la dinámica energética y a las entradas y salidas de agua del balance hídrico.

El contenido hídrico del suelo respondió de forma positiva a las cantidades de precipitación y de forma negativa a la demanda evapotranspirativa, lo que permitió diferenciar cuatro periodos principales (Figura $\mathrm{N}^{\circ} 4$ ):

- un periodo de humectación coincidente con otoño, que puede ser más o menos pronunciado debido a la variabilidad de la lluvia y a la, todavía, alta evapotranspiración.

- un periodo húmedo, coincidente con los meses de invierno, en el que el contenido de agua normalmente alcanza sus valores estacionales más altos e incluso la saturación, debido a la baja demanda evapotranspirativa y a la sucesión de las precipitaciones.

- un periodo de secado que normalmente tiene lugar en primavera, caracterizado por el progresivo incremento de la demanda evapotranspirativa y una gran irregularidad pluviométrica, que resulta en una tendencia decreciente de la humedad con fuertes oscilaciones puntuales durante el descenso.

- un periodo estival, correspondiente con el verano, caracterizado por registrar los contenidos hídricos del suelo más bajos, y que tiene su origen en la falta de precipitaciones y la fuerte demanda evapotranspirativa.

Resultados similares fueron observados por Martínez-Fernández \&Ceballos-Barbancho (2003), Cubera \&Moreno (2007) o García-Estringana et al. (2013) en ambientes mediterráneos de la Península Ibérica. Dichos autores observaron patrones estaciones en la dinámica hídrica del suelo asociados a las precipitaciones y a la demanda evaporativa. Por otro lado, los contenidos hídricos del suelo presentaron diferente respuestas ante los periodos mencionados según la profundidad considerada, indicando que la capa superficial del suelo (primeros $15 \mathrm{~cm}$ ) fue la más expuesta a los factores ambientales externos (Lozano-Parra \& Schnabel, 2015, Lozano-Parra et al., 2016, Schnabel et al., 2018). 
Figura $\mathrm{N}^{\circ} 4$

Dinámica hídrica del suelo a escala diaria y a diferentes profundidades durante el periodo vegetativo de las herbáceas anuales, en una estación de humedad ubicada bajo copa de árbol

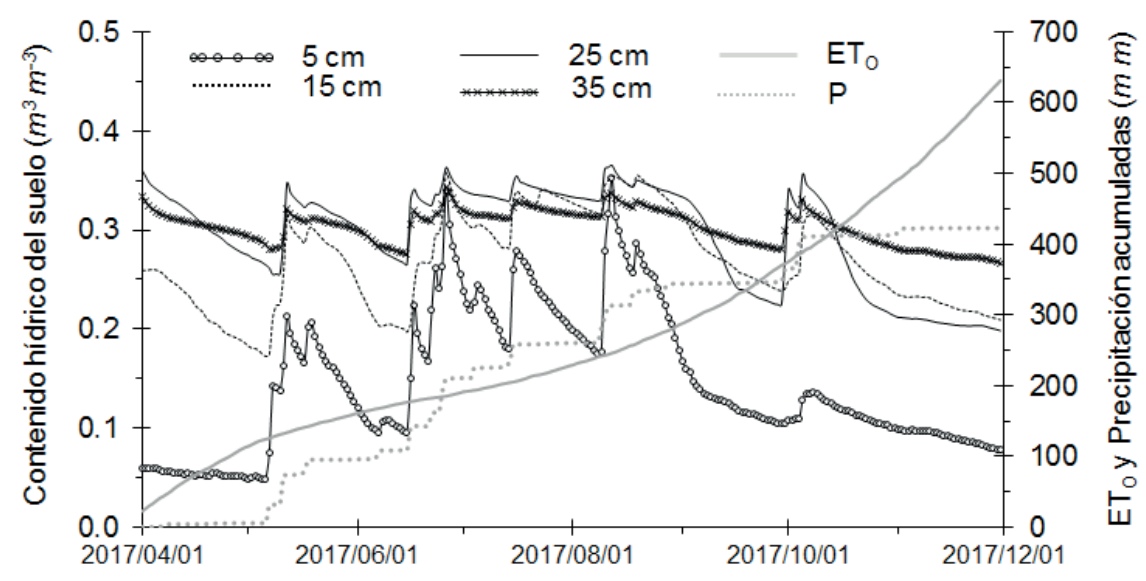

Fuente: Elaboración propia.

Las diferencias relativas en los contenidos hídricos del suelo entre EHS durante el ciclo vital de las herbáceas anuales se presentan en la Figura $N^{\circ} 5$. En una inspección inicial, se observan dos EHS de distintas coberturas (P3 y A1) que registraron mayores contenidos, mientras que el resto registró valores similares o inferiores al promedio relativo del área de estudio. De esta forma, determinar un patrón de comportamiento hídrico que indique qué cobertura vegetal (arbolado o pastizal) registra mayores cantidades de agua fue complejo.

Figura $\mathrm{N}^{\circ} 5$

Diferencias relativas (\%) en los contenidos hídricos del suelo entre las estaciones de humedad instaladas en pastizal $(P)$ y bajo copa de árbol $(A)$ durante el ciclo de vida de las herbáceas anuales (otoño, invierno y primavera)

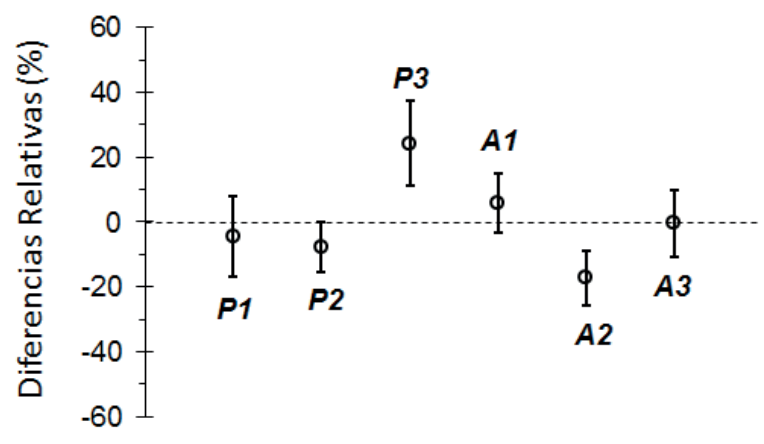

Fuente: Elaboración propia. 
Con el fin de establecer las diferencias estadísticas entre las EHS, se aplicó el test no-paramétrico de Kruskal-Wallis (Cuadro $\mathrm{N}^{\circ} 3$ ), que fue realizado tras contrastar, mediante el test de Shapiro-Wilk, que la distribución de los contenidos hídricos diarios en las EHS no fue normal. Los resultados mostraron diferencias entre las EHS de distintas coberturas, excepto entre A3 y las estaciones de pastizal P1 y P2 (Cuadro $N^{\circ} 3$ ). Resultados similares fueron observados por Gea-Izquierdo et al. (2009), Lozano-Parra et al. (2011) o Lozano-Parra et al. (2015) en sistemas silvopastoriles mediterráneos de la Península Ibérica, quienes concluyen que establecer un patrón de comportamiento hídrico entre diferentes coberturas vegetales no debería ser generalizado, ya que el efecto de la cobertura sobre la humedad del suelo parece ser variable en función de factores como las características climáticas del año hidrológico considerado, la fenología y morfología vegetal, las propiedades edáficas, o la ubicación relativa de la vegetación sobre la cuenca hidrológica.

\section{Cuadro $\mathrm{N}^{\circ} 3$}

Test no-paramétrico Kruskal-Wallis ANOVA entre las estaciones de humedad del suelo (EHS). $\mathrm{P}=$ Pastizal; $\mathrm{A}=$ Árbol; $\mathrm{n} . \mathrm{s}=$ no significativo; ${ }^{*}=p<0.05 ;{ }^{* *}=p<0.01$.

\begin{tabular}{|l|r|r|r|r|r|}
\hline & P1 & P2 & P3 & A1 & A2 \\
\hline P2 & n.s & & & & \\
\hline P3 & $* *$ & $* *$ & & & \\
\hline A1 & $* *$ & $* *$ & $* *$ & & \\
\hline A2 & n.s & n.s & $* *$ & $* *$ & $* *$ \\
\hline A3 & n.s & $* *$ & $*$ & $*$ \\
\hline
\end{tabular}

Fuente: Elaboración propia.

\section{Crecimiento de la biomasa herbácea entre distintas coberturas y NDVI}

El crecimiento de la biomasa herbácea mostró dependencia de la variabilidad en la disponibilidad hídrica del suelo (Figura $N^{\circ} 6$ ). A principios de otoño, la vegetación registró valores mínimos de 0.1 en el NDVI, próximos a los valores de suelo desnudo. El incremento de los contenidos hídricos del suelo y la disminución de los potenciales de succión provocaron una respuesta directa en el desarrollo de las herbáceas, ya que la reserva hídrica se encontró más fácilmente disponible para el consumo vegetal. No obstante, el crecimiento de las herbáceas se estancó entre mediados de junio y finales de julio, probablemente debido a los condicionantes energéticos impuestos por el clima (luz y calor). Desde agosto se apreció un desarrollo sostenido, posiblemente debido a cambios en el balance energético (Figura $\mathrm{N}^{\circ} 6$ ).

El crecimiento de la biomasa aérea fue utilizado como variable indicadora del comportamiento del NDVI. Para ello se estableció la relación entre las mediciones del pasto en altura $(\mathrm{cm})$ y el 
Figura $N^{\circ} 6$

Dinámica de la humedad del suelo $(\theta)$, potencial hídrico del suelo $(\Psi)$ e Índice Diferencial de Vegetación Normalizado (NDVI) registrado en pastizal. Los valores representan datos en intervalos diarios durante el ciclo de vida de las herbáceas anuales

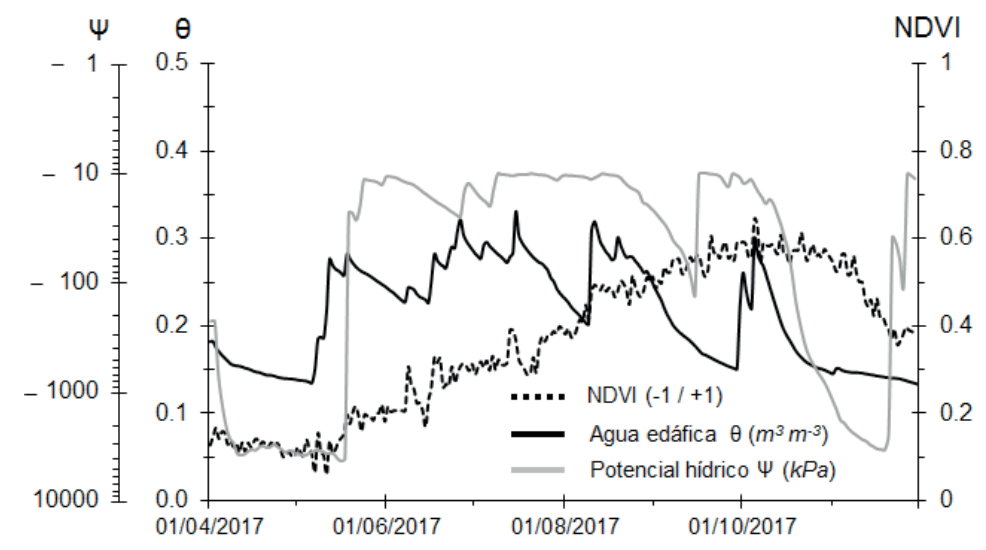

Fuente: Elaboración propia.

NDVI (Figura $\mathrm{N}^{\circ} 7$ ). El resultado muestra una curva exponencial bastante ajustada para la biomasa creciendo bajo los dos tipos de coberturas vegetales analizadas, en espacios abiertos y bajo copa de árbol (Figura $\mathrm{N}^{\circ} 7$ ), indicando que el NDVI captura correctamente las fases en el crecimiento vegetal y que las mediciones en altura del crecimiento de la biomasa suponen un método alternativo adecuado para monitorizar la vegetación con una metodología no destructiva. Los valores más bajos corresponden con una vegetación incipiente que comienza a brotar a finales de otoño, mientras que los más altos se corresponden con situaciones de mediados-finales de primavera. Las alturas más elevadas registradas bajo copa de árbol podrían indicar una mayor producción de biomasa herbácea bajo esta cobertura vegetal que en los espacios abiertos. Resultados similares también fueron observados por Masková et al. (2008) o Rahetlah et al. (2014), quienes observaron estrechas relaciones en diferentes agrosistemas entre las mediciones de biomasa en altura y el NDVI. Cabe mencionar que altos valores de NDVI están relacionados con mayores producciones de biomasa herbácea, como ya ha sido observado por Flynn et al. (2006) o, más recientemente, por Schucknecht et al. (2017). De este modo, se intentó establecer la pro-

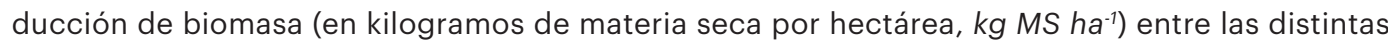
coberturas. Para ello, se utilizaron las relaciones alométricas entre altura de las herbáceas y producción de biomasa definidas por Lozano-Parra et al. (2014) y Lozano-Parra \&Schnabel (2015). Las estimaciones mostraron que los espacios bajo la copa del árbol pueden alcanzar una producción de $1938 \mathrm{~kg} \mathrm{MS} \mathrm{ha-1}^{-1}$, mientras que en los espacios abiertos pueden obtenerse $1106 \mathrm{~kg}$ MS ha-1. Los efectos positivos de los árboles sobre la producción de biomasa han sido reportados por muchos autores. Por ejemplo, Moreno et al. (2013), mientras que resultados opuestos se pueden encontrar en estudios de revisión como Marañón et al. (2009). La productividad de los sistemas silvopastoriles mediterráneos con cobertura arbórea dispersa puede presentar, por lo tanto, gran incertidumbre y variaciones a diferentes escalas espaciales y temporales. 
Figura $\mathrm{N}^{\circ} 7$

Relación entre el crecimiento de las herbáceas y el Índice Diferencial de Vegetación Normalizado (NDVI).

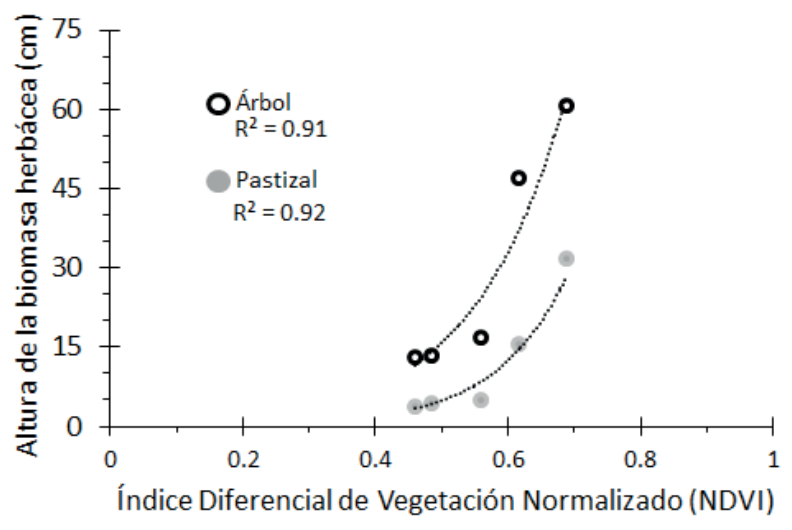

Fuente: Elaboración propia.

\section{El papel del agua en el crecimiento de la biomasa herbácea}

Con el fin de establecer la relación entre los flujos hídricos (en varias etapas del ciclo del agua, como la precipitación, evapotranspiración, o contenido hídrico del suelo) y el crecimiento de la biomasa herbácea, se llevó a cabo un análisis de correlación no-paramétrico entre las variables diarias asociadas al ciclo hidrológico y la dinámica vegetal diaria monitorizada a través del NDVI (Cuadro $\mathrm{N}^{\circ} 4$ ). Los resultados muestran una relación significativa entre dinámica de la biomasa (NDVI) y la evapotranspiración. Esto puede explicarse porque la transpiración, fenómeno asociado a la pérdida de agua en la planta por su actividad fotosintética y desarrollo, es esencialmente el mismo proceso físico que la evaporación (Dingman, 2015). Por ello, un aumento en la evapotranspiración podría conllevar, hasta cierto punto, un aumento en la biomasa herbácea. No obstante, variables como el contenido hídrico del suelo o la precipitación no mostraron correlaciones satisfactorias con el NDVI (Cuadro $\mathrm{N}^{\circ} 4$ ), lo que podría indicar, por un lado, que tienen una incidencia positiva sobre la biomasa cuando sus efectos son analizados de forma acumulada sobre el tiempo y, por otro, que no interaccionan por sí solas con la dinámica vegetal, sino que lo hacen de forma combinada junto con otras variables, como por ejemplo las asociadas a la disponibilidad energéti$c a$, que se ven reflejadas en la temperatura ambiente o, indirectamente, en la evapotranspiración.

Debido a que los factores individuales que controlan la dinámica de la biomasa herbácea interactúan entre ellos y en el tiempo, se llevó a cabo un análisis de regresión múltiple, con el fin de considerar el efecto combinado de las variables asociadas al ciclo hidrológico sobre la dinámica de la biomasa herbácea. Los resultados, presentados el Cuadro $N^{\circ} 5$, demostraron que entre el $57 \%$ y el $93 \%$ de la varianza en la dinámica del NDVI puede ser explicada utilizando únicamente tres variables asociadas al ciclo hidrológico, es decir, a partir del contenido hídrico del suelo, la precipitación y la evapotranspiración. Sin embargo, los análisis estadísticos mejoraron cuando estas dos últimas variables fueron incluidas de forma acumulada sobre el tiempo. Esta situación 


\section{Cuadro $\mathrm{N}^{\circ} 4$}

Test no-paramétrico de correlación de Spearman. ET = Evapotranspiración; $\mathrm{P}=$ Precipitación;

ET Acum. = Evapotranspiración acumulada; $\mathrm{P}$ Acum $=$ Precipitación acumulada. ${ }^{*}=p<0.05$

\begin{tabular}{|l|r|r|r|r|r|r|}
\hline & \multicolumn{1}{|c|}{$\begin{array}{c}\text { Agua } \\
\text { suelo }\end{array}$} & $\begin{array}{c}\text { Potencial } \\
\text { succión }\end{array}$ & ET & P & $\begin{array}{c}\text { ET } \\
\text { Acum. }\end{array}$ & $\begin{array}{c}\text { P } \\
\text { Acum. }\end{array}$ \\
\hline Potencial succión & ${ }^{*} 0.95$ & & & & & \\
\hline ET & ${ }^{*}-0.71$ & ${ }^{*}-0.68$ & & & & \\
\hline$P$ & ${ }^{*} 0.34$ & ${ }^{*} 0.29$ & ${ }^{*}-0.31$ & & & \\
\hline ET Acum. & ${ }^{*}-0.20$ & -0.12 & ${ }^{*} 0.55$ & -0.03 & & \\
\hline P Acum. & ${ }^{*}-0.19$ & -0.12 & ${ }^{*} 0.55$ & -0.02 & ${ }^{*} 1.00$ & \\
\hline NDVI & 0.02 & -0.01 & ${ }^{*} 0.43$ & 0.07 & ${ }^{*} 0.86$ & ${ }^{*} 0.86$ \\
\hline
\end{tabular}

Fuente: Elaboración propia.

pone de manifiesto la complejidad en la dinámica de la biomasa para ser explicada a partir de las variables hidrológicas, y enfatiza los efectos retardados que caracterizan a los procesos que ocurren en los ecosistemas, en este caso los procesos de retroalimentación entre las entradas y salidas de agua en el sistema y la respuesta vegetal.

Si los patrones del ciclo hidrológico se modifican y los episodios secos se vuelven más frecuente en el presente siglo debido a un cambio climático (IPCC, 2013), una importante superficie podría ser menos productiva, considerando que los sistemas silvopastoriles de espinal ocupan una área muy extensa.

\section{Cuadro $N^{\circ} 5$}

Análisis de regresión múltiple sobre los factores que influyen en la dinámica del NDVI. $\theta=$ contenido hídrico del suelo ET = Evapotranspiración; $\mathrm{P}=$ Precipitación; $\mathrm{ET}$ Acum. $=$ Evapotranspiración acumulada; P Acum. $=$ Precipitación acumulada. ${ }^{*}=p<0.05$

\begin{tabular}{|c|c|c|c|c|}
\hline Modelo & $\mathrm{R}^{2}$ & \multicolumn{2}{|c|}{$\beta$} & $p$ \\
\hline \multirow{4}{*}{$N D V I=\beta_{a}+\theta * \beta_{b}+E T * \beta_{c}+P * \beta_{d}$} & \multirow{4}{*}{0.57} & a & -0.261783 & 0.00 \\
\hline & & $\mathrm{b}$ & 1.710360 & 0.00 \\
\hline & & $\mathrm{C}$ & 0.102997 & 0.00 \\
\hline & & d & 0.001167 & 0.44 \\
\hline \multirow{4}{*}{ NDVI $=\beta_{\mathrm{a}}+\theta{ }^{*} \beta_{\mathrm{b}}+\mathrm{ET}$ Acum. ${ }^{*} \beta_{\mathrm{c}}+\mathrm{P}$ Acum. ${ }^{*} \beta_{\mathrm{d}}$} & \multirow{4}{*}{0.93} & $\mathrm{a}$ & 0.269841 & 0.00 \\
\hline & & $\mathrm{b}$ & 0.001888 & 0.00 \\
\hline & & $\mathrm{c}$ & -0.000933 & 0.00 \\
\hline & & $d$ & -0.601144 & 0.00 \\
\hline
\end{tabular}

Fuente: Elaboración propia. 


\section{Consideraciones finales}

Este estudio destaca el importante papel que desempeña el agua sobre la dinámica de la biomasa herbácea en los ecosistemas de espinal con clima mediterráneo de la zona central de Chile.

Los resultados mostraron que las variables asociadas al ciclo hidrológico, como la precipitación, evapotranspiración y contenido hídrico del suelo, presentaron dificultades para explicar por sí solas la dinámica de la biomasa herbácea. Sin embargo, cuando estas variables fueron consideradas de forma combinada, su capacidad para explicar dicha dinámica aumentó entre un 57\% y $93 \%$. Asimismo, las mediciones del crecimiento de la biomasa herbácea en altura demostraron ser una adecuada metodología para monitorizar la dinámica del NDVI de forma no destructiva.

Por otro lado, establecer un patrón de comportamiento hídrico del suelo entre diferentes coberturas vegetales (bajo la copa de árbol y fuera de su influencia) fue complejo, ya que el efecto de la cobertura sobre la humedad edáfica puede variar en función de factores como las características climáticas del año hidrológico, la fenología y morfología vegetal, las propiedades edáficas, o la ubicación relativa de la vegetación sobre la cuenca hidrológica.

Considerando la amplitud de los sistemas silvopastoriles de espinal, una superficie importante podría ser menos productiva si los patrones del ciclo hidrológico se modificaran debido a un cambio climático. Con el fin de mejorar la comprensión sobre el funcionamiento de estos ecosistemas y poder aplicar medidas de gestión del territorio más eficientes, se requiere más investigación desde un enfoque ecohidrológico en los sistemas silvopastoriles de espinal.

\section{Referencias bibliográficas}

ALLEN, R.G.; PEREIRA, L.S.; RAES, D. \& SMITH, M. Crop evapotranspiration - Guidelines for computing crop water requirements - FAO Irrigation and drainage paper 56. Rome: FAO - Food and Agriculture Organization of the United Nations, 1998.

BROOKER, R.W.; MAESTRE, F.T.; CALLAWAY, R.M.; LORTIE, C.L.; CAVIERES, L.A.; KUNSTLER, G.; LIANCOURT, P.; TIELBÖRGER, K.; TRAVIS, J.M.J.; ANTHELME, F.; ARMAS, C.; COLL, L.; CORCKET, E.; DELZON, S.; FOREY, E.; KIKVIDZE, Z.; OLOFSSON, J.; PUGNAIRE, F.; QUIROZ, C.L.; SACCONE, P.; SCHIFFERS, K.; SEIFAN, M.; TOUZARD, B. \& MICHALET, R. Facilitation in plant communities: the past, the present, and the future. Journal of Ecology, 2008, N 96, p. 18-34.

CAMPOS-PALACÍN, P. Towards a sustainable global economics approach for Mediterranean agroforestry systems. In: SCHNABEL, S. \& FERREIRA, A. Sustainability of agrosilvopastoral systems -Dehesas, Montados-. Advances in Geoecology. Reiskirchen: Catena Verlag, 2004, p. 13-28.

CAMPOS, P.; HUNTSINGER, L.; OVIEDO, J.L.; STARRS, P.F.; DIAZ, M.; STANDIFORD, R.B. \& MONTERO, G. Mediterranean Oak Woodland Working Landscapes. Dehesas of Spain and Ranchlands of California. Springer Netherlands, 2013. 
CANADELL, J. \& ZEDLER, P.H. Underground structures of woody plants in Mediterranean ecosystems of Australia, California, and Chile. In: KALIN, M.T.; ZEDLER, P.H. \& FOX, M.D. Ecology and biogeography of Mediterranean ecosystems in Chile, California and Australia. New York: Springer-Verlag, 1995, p. 177-210.

CHUVIECO, E. Fundamentals of Satellite Remote Sensing: An Environmental Approach. USA: CRC Press, 2016.

CUBERA, E. \& MORENO, G. Effect of single Quercus ilex trees upon spatial and seasonal changes in soil water content in dehesas of central western Spain. Annals of Forest Science, 2007, № 64, p. 355-364.

DINGMAN, S. L. Physical Hydrology. Third Edition. USA: Waveland Press, Inc, 2015.

FOOD AND AGRICULTURE ORGANIZATION (FAO). Harmonized World Soil Database. FAO/IIASA/ ISRIC/ISS-CAS/JRC, 2012, No V 1.2, p. Viewer version.

FLYNN, E.; DOUGHERTY, C. \& WENDROTH, O. Assessment of Pasture Biomass with the Normalized Difference Vegetation Index from Active Ground-Based Sensors. Agronomy Journal, 2006, № 100, p. 114-121.

GARCÍA-ESTRINGANA, P.; LATRON, J.; LLORENS, P. \& GALLART, F. Spatial and temporal dynamics of soil moisture in a Mediterranean mountain area (Vallcebre, NE Spain). Ecohydrology, 2013, $\mathrm{N}^{\circ}$ 6, p. 741-753.

GARREAUD, R.D.; VUILLE, M.; COMPAGNUCCI, R. \& MARENGO, J. Present-day south american climate. Palaeogeography, Palaeoclimatology, Palaeoecology, 2009, № 281, p. 180-195.

GEA-IZQUIERDO, G.; MONTERO, G. \& CAÑELLAS, I. Changes in limiting resources determine spatio-temporal variability in tree-grass interactions. Agroforestry Systems, 2009, No 76, p. 375-387.

IPCC. Climate Change 2013. Summary for Policymakers. In: STOCKER, T.F., QIN, D.; PLATTNER, G.-K.; TIGNOR, M.M.B.; ALLEN, S.K.; BOSCHUNG, J.; NAUELS, A.; XIA, Y.; BEX, V. \& MIDGLEY, P. M. Climate Change 2013: The Physical Science Basis. Contribution of Working Group I to the Fifth Assessment Report of the Intergovernmental Panel on Climate Change. United Kingdom and New York, NY, USA: Cambrige University Press, 2013, p. 33 p.

LOZANO-PARRA, J. Dinámica del agua edáfica en dehesas y su relación con el clima y la vegetación. Boletín de la Asociación de Geográfos Españoles, 2015, № 69, p. 625-629.

LOZANO-PARRA, J.; MANETA, M. \& SCHNABEL, S. Climate and topographic controls on simulated pasture production in a semiarid Mediterranean watershed with scattered tree cover. Hydrology and Earth System Sciences, 2014, № 18, p. 1439-1456.

LOZANO-PARRA, J. \& SCHNABEL, S. Respuesta de la vegetación herbácea a las variaciones hídricas del suelo. En: MARTíNEZ PÉREZ, S. y SASTRE MERLíN, A. Estudios de la Zona No Saturada. Alcalá de Henares: Universidad de Alcalá de Henares, 2015, p. 77-84. 
LOZANO-PARRA, J.; SCHNABEL, S. y CEBALLOS-BARBANCHO, A. Dinámica del agua del suelo en dehesa bajo diferentes cubiertas vegetales. Resultados preliminares. En: MARTíNEZ-FERNÁNDEZ, J. y SANCHEZ MARTíN, N. Estudios de la Zona No Saturada del Suelo. Salamanca: Universidad de Salamanca, 2011, p. 47-52.

LOZANO-PARRA, J.; SCHNABEL, S. \& CEBALLOS-BARBANCHO, A. The role of vegetation covers on soil wetting processes at rainfall event scale in scattered tree woodland of Mediterranean climate. Journal of Hydrology, 2015, № 529, p. 951-961.

LOZANO-PARRA, J.; VAN SCHAIK, L.; SCHNABEL, S. \& GÓMEZ-GUTIÉRREZ, Á. Soil moisture dynamics at high temporal resolution in a mediterranean watershed with scattered tree cover. Hydrological Processes, 2016, №30, p. 1155-1170.

MARAÑÓN, T., PUGNAIRE, F. \& CALLAWAY, R. M. Mediterranean-climate oak savannas: the interplay between abiotic environment and species interactions. Web Ecology, 2009, N 9, p. 30-43. MARTÍNEZ-FERNÁNDEZ, J. \& CEBALLOS-BARBANCHO, A. Temporal stability of soil moisture in a large-field experiment in Spain. Soil Science Society of America, 2003, № 67, p. 1647-1656.

MARTÍNEZ-FERNÁNDEZ, J. \& CEBALLOS BARBANCHO, A. Mean soil moisture estimation using temporal stability analysis. Journal of Hydrology, 2005, N 312, p. 28-38.

MASKOVÁ, Z.; ZEMEK, F. \& KVET, J. Normalized difference vegetation index (NDVI) in the management of mountain meadows. Boreal Environment Research, 2008, N 13, p. 417-432.

MORENO, G. Response of understorey forage to multiple tree effects in Iberian dehesas. Agriculture, Ecosystems and Environment, 2008, №123, p. 239-244.

MORENO, G.; BARTOLOME, J.W.; GEA-IZQUIERDO, G. \& CAÑELLAS, I. Overstory-Understory Relationships. En: CAMPOS, P.; HUNTSINGER, L.; OVIEDO, J.L.; STARRS, P.F.; DÍAZ, M.; STANDIFORD, R.B. \& MONTERO, G. Mediterranean oak woodland working landscapes. Dehesas of Spain and Ranchlands of California. Netherlands: Springer, 2013, p. 508.

OVALLE, C.; ARONSON, J.; DEL POZO, A. \& AVENDANO, J. The espinal: agroforestry systems of the mediterranean - type climate region of Chile. Agroforestry Systems, 1990, No 10, p. 213-239.

RAHETLAH, V.; SALGADO, P.; ANDRIANARISOA, B.; TILLARD, B.; RAZAFINDRAZAKA, H.; MÉZO, L. \& RAMALANJAONA, V. Relationship between normalized difference vegetation index (NDVI) and forage biomass yield in the Vakinankaratra region, Madagascar. Livestock Research for Rural Development, 2014, $\mathrm{N}^{\circ} 26$, p. 1-11.

REYNOLDS, J.F.; SMITH, D.M.S.; LAMBIN, E.F.; TURNER, B.L.; MORTIMORE, M.; BATTERBURY, S.P.J.; DOWNING, T.E.; DOWLATABADI, H.; FERNANDEZ, R.J.; HERRICK, J. E.; HUBER-SANNWALD, E.; JIANG, H.; LEEMANS, R.; LYNAM, T.; MAESTRE, F.T.; AYARZA, M. \& WALKER, B. Global Desertification: Building a Science for Dryland Development. Science, 2007, N³16, p. 847-851.

RIGUEIRO-RODRÍGUEZ, A.; MCADAM, J. \& MOSQUERA-LOSADA, M. R. Agroforestry in Europe: Current Status and Future Prospects. Netherlands: Springer, 2009. 
ROOT-BERNSTEIN, M. \& JAKSIC, F. The Chilean Espinal: Restoration for a Sustainable Silvopastoral System. Restoration Ecology, 2013, N²1, p. 409-414.

RUBEL, F. \& KOTTEK, M. Observed and projected climate shifts 1901-2100 depicted by world maps of the Koppen-Geiger climate classification. Meteorologische Zeitschrift, 2010, № 19, p. 135-141.

SCHNABEL, S.; DAHLGREN, R.A. \& MORENO, G. Soil and water dynamics. En: CAMPOS, P.; HUNTSINGER, L.; OVIEDO, J.L.; STARRS, P.F.; DÍAZ, M.; STANDIFORD, R. \& MONTERO, G. Mediterranean oak woodland working landscapes. Dehesas of Spain and ranchlands of California. New York: Springer-Verlag, 2013, p. 91-121.

SCHNABEL, S.; GONZÁLEZ, F.; MURILLO, M. \& MORENO, V. Different techniques of pasture improvement and soil erosion in a Wooded Rangeland in SW Spain: methodology and preliminary results. In: CONACHER, A. J. Land Degradation. Dordrecht: Kluwer Academics, 2001, p. 239-253.

SCHNABEL, S.; LOZANO-PARRA, J.; GÓMEZ-GUTIÉRREZ, Á. \& ALFONSO-TORREÑO, A. Hydrological dynamics in a small catchment with silvopastoral land use in SW Spain. Cuadernos de Investigación Geográfica, 2018, № 44, p. 1-19.

SCHUCKNECHT, A.; MERONI, M.; KAYITAKIRE, F. \& BOUREIMA, A. Assessment of Pasture Biomass with the Normalized Difference Vegetation Index from Active Ground-Based Sensors. Remote Sensing, 2017, N 9, p. 463.

VACHAUD, G.; PASSERAT DE SILANS, A.; BALABANIS, P. \& VAUCLIN, M. Temporal stability of spatially measured soil water probability density function. Soil Science Society of America Journal, $1985, N^{\circ} 49$, p. 822-828.

VALDÉS-PINEDA, R.; PIZARRO, R.; GARCÍA-CHEVESICH, P.; VALDÉS, J.; OLIVARES, C.; VERA, M.; BALOCCHI, F.; PÉREZ, F.; VALLEJOS, C.; FUENTES, R.; ABARZA, A. \& HELWIG, B. Water governance in Chile: Availability, management and climate change. Journal of Hydrology, 2014, $N^{\circ} 519, p$. 2538-2567.

VALLADARES, F.; VILAGROSA, A.; PEÑUELAS, J.; OGAYA, R.; CAMARERO, J.J.; CORCUERA, L.; SISÓ, S. \& GIL-PELEGRÍN, E. Estrés hídrico: ecofisiología y escalas de sequía. En: VALLADARES, F. Ecología del bosque mediterráneo en un mundo cambiante. Ministerio de Medio Ambiente. EGRAF, S.A., 2004, p. 165-192. 